\title{
Microbial and nutritional influence on endocrine control of growth
}

\author{
Filipe De Vadder, Amélie Joly and François Leulier
}

Institut de Génomique Fonctionnelle de Lyon, Université de Lyon, École Normale Supérieure de Lyon, Centre National de la Recherche Scientifique, Université Claude Bernard Lyon 1, Lyon, France

Correspondence should be addressed to F De Vadder or F Leulier: filipe.de_vadder@ens-lyon.fr or francois.leulier@ens-lyon.fr

This paper is part of a collection of articles exploring Gut Microbiome and Endocrinology, across the Journal of Endocrinology and the Journal of Molecular Endocrinology. The editor for this section was Dr Jonathon Schertzer.

\begin{abstract}
The worrying number of children suffering from undernutrition and consequent stunting worldwide makes the understanding of the relationship between nutritional status and postnatal growth crucial. Moreover, it is now well established that undernourished children harbor an altered microbiota, correlating with impaired growth. In this review, we describe how murine models have been used to explore the functional relationships between endocrine regulation of growth, nutrition and gut microbiota. In numerous Mammalian species, postnatal growth is mainly regulated by the conserved GH/IGF1 somatotropic axis that acts through endocrine and paracrine pathways, notably enabling longitudinal bone growth. Recent studies have demonstrated that the microbiota effects on growth could involve a modulation of GH and IGF1 circulating levels. Besides, the GH/IGF1 somatotropic axis may regulate the gut microbiota composition and diversity. Studying the bidirectional relationship between growth hormones and the gut microbiome could therefore help developing microbiota-targeting therapies in order to reduce the long-term consequences of stunting.
\end{abstract}
Key Words
- microbiome
- IGF
- growth factors
- linear growth

\section{Introduction}

The growth hormone (GH)/insulin-like growth factor-1 (IGF1) somatotropic axis plays a crucial role in the regulation of growth. $\mathrm{GH}$ is known for its anabolic effects on the intestine, stimulation of catabolism in adipose tissue, as well as bone growth (Jensen et al. 2020b). Effects of GH occur independently or in synergy with IGF1 (Yakar \& Isaksson 2016, Poinsot et al. 2018, Jensen et al. $2020 b$ ). Interestingly, circulating levels of IGF1 depend on the nutritional status of the organism. Indeed, during an acute phase of protein malnutrition, resulting in stunting, IGF1 secretion is strongly diminished (Fazeli \& Klibanski 2014, Schwarzer et al. 2016).

In humans, as defined by the World Health Organization, undernutrition triggers stunting (low height-for-age), wasting (low weight-for-height) or underweight (low weight-for-age) (https:// globalnutritionreport.org/reports/2020-global-nutritionreport/). Undernutrition has been associated to alterations in the gut microbial communities, correlating with poor growth patterns (Subramanian et al. 2014). Moreover, colonization of mouse and pig gnotobiotic models with the microbiota of human undernourished patients reproduces the wasting and stunting phenotype observed in humans (Smith et al. 2013, Blanton et al. 2016b, Gehrig et al. 2019). Although the exact mechanisms underlying such phenotypes remain to be elucidated, expression levels of proteins involved in the GH/IGF1 axis strongly correlate with length-for-age $\mathrm{z}$ score (Chen et al. 2020).

In the past 15 years, the role of the gut microbiota has been widely studied, expanding into the understanding 
of its impact on physiological processes such as growth, endocrine function and regulation of host metabolism. In this review, we explore associations between the gut microbiota, GH/IGF1 somatotropic axis and systemic growth in mammals. Furthermore, we provide insight into the use of selected microbial strains for the management of undernutrition-related diseases.

\section{Gut microbiota and regulation of mammalian growth}

\section{Involvement of the GH/IGF1 axis in growth}

The mammalian somatotropic axis is centrally regulated by $\mathrm{GH}$, which is a 191-amino acid peptide secreted by the anterior pituitary gland. In the intestine, GH activates its receptor (GHR) (Delehaye-Zervas et al. 1994), inducing proliferation of intestinal stem cells and improving nutrient absorption (Young et al. 2019). In the bone, GH regulates chondrocyte multiplication and hypertrophy (Mackie et al. 2011). While GH directly influences the growth of several organs (Jensen et al. 2020b), its most significant effects on growth are obtained by enhancing the production of IGF1, a potent growth factor that stimulates growth and metabolism (Yakar \& Isaksson 2016). GH secretion is promoted by GH releasing hormone, ghrelin and leptin, while being inhibited by IGF1 and somatostatin (Burgus et al. 1973, Carro et al. 1997, Kojima et al. 1999) (Fig. 1). Somatostatin, ghrelin and leptin are also known for their roles in appetite regulation and their secretion has been linked to the gut microbiota (Karsenty 2006, Pradhan et al. 2013).

IGF1 acts via a receptor which is widely expressed in most cell types (excepted hepatocytes) (Bartke et al. 2013), leading to the transcription of target genes (Papaconstantinou 2009). IGF1 is essential for growth at two periods: during the embryonic development in a GH-independent manner and during childhood and adolescence in a GH-dependent manner (Netchine et al. 2011). Stimulation of IGF1 production by GH elicits growth in two different ways. Indeed, liver-produced IGF1 can act as a hormone, while peripheral production leads to autocrine or paracrine signaling (Mohan \& Kesavan 2012). IGF1 stimulates skeletal muscle mass development (Sharples et al. 2015) and bone growth by both pathways, since liver-specific deletion of $I g f 1$ (decreasing circulating

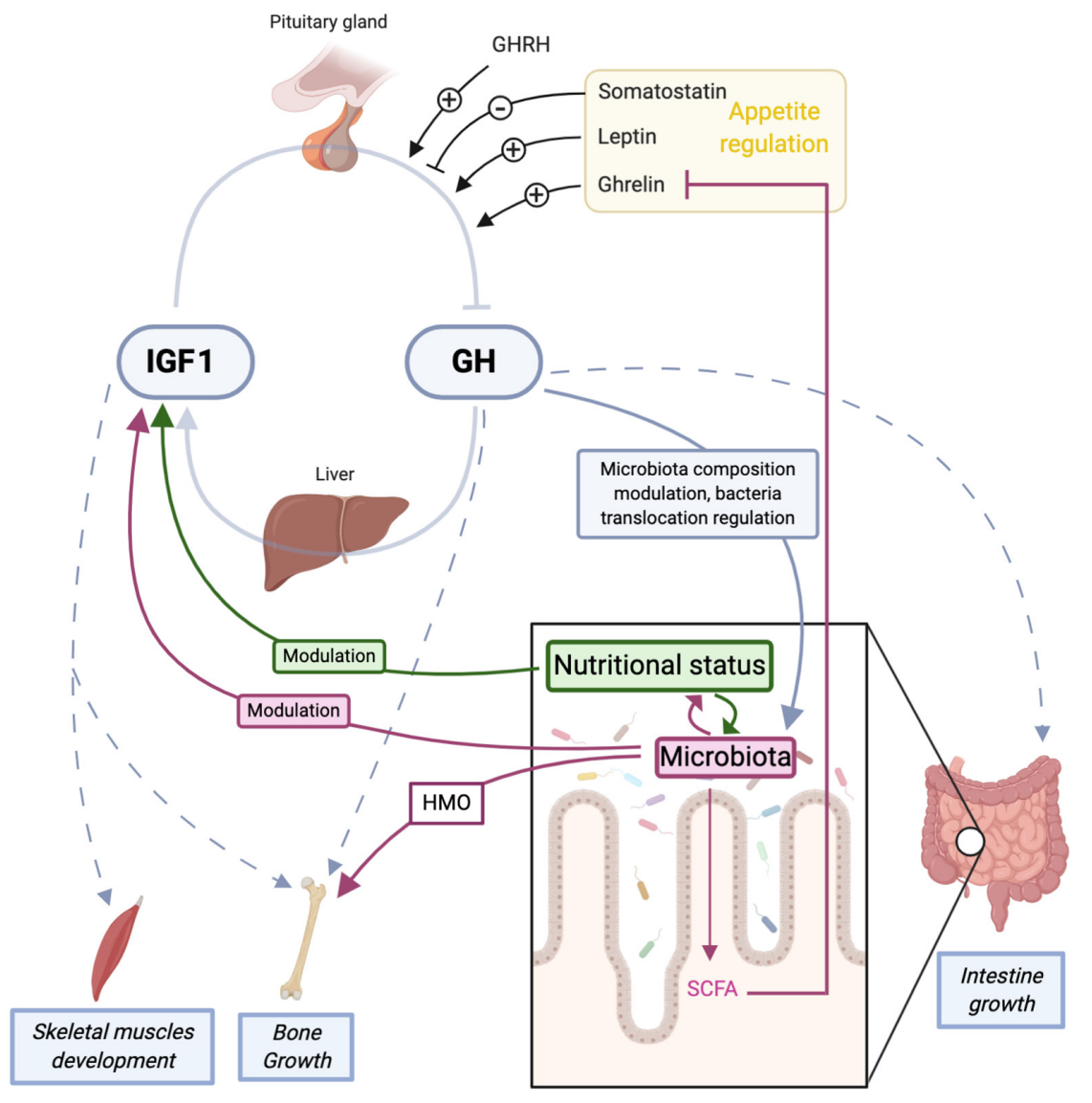

\section{Figure 1}

The reciprocal relationship between the GH/IGF1 somatotropic axis, the gut microbiota and the nutritional status modulates systemic growth. GH (growth hormone) secretion in the pituitary gland is enhanced by GHRH (GH releasing hormone), leptin and ghrelin and inhibited by somatostatin. GH notably promotes intestine and bone growth and promotes IGF1 production in the liver and in other peripheral organs (not shown). IGF1 in turn reduces $\mathrm{GH}$ secretion and activates bone growth and skeletal muscle development. In the intestine, the microbiota can modulate IGF1 secretion and therefore bone growth. A possible mechanism for this modulation involves ghrelin inhibition by microbiota-derived short chain fatty acids (SCFA). Microbiota composition is also impacted by the nutritional status (e.g. undernutrition), which also affects IGF1 production. In turn, circulating GH modulates microbiota composition as well as bacteria translocation through the intestinal epithelium. Beyond the GH/IGF1 axis, microbiota could modulate systemic growth by interacting with human milk oligosaccharides (HMO). Created with biorender.com. 
IGF1 by 70\%) does not impair growth in the first weeks of life (Yakar et al. 2002).

IGF1 genetic deficiency is a rare autosomal recessive mutation but, in cases where it has been described, it leads to pre- and postnatal growth retardation and microcephaly. Patients with the mutation can benefit from recombinant IGF1. These observations show that IGF1 signaling is crucial for optimal growth and CNS development.

\section{Gut microbiota influence on GH and IGF1}

Interestingly, germ-free mice, which lack a gut microbiota, have impaired growth (Schwarzer et al. 2016, Yan et al. 2016, Novince et al. 2017). Previous work from our lab has shown that germ-free mice have reduced circulating IGF1 and insulin-like growth factor binding protein-3 (IGFBP3, IGF1 major binding protein), as well as reduced $I g f 1$ and Igfbp3 expression in the liver (Schwarzer et al. 2016). Specifically, when germ-free mice were undernourished with a protein- and fat-depleted diet, they exhibit stronger stunting phenotype than their conventional counterparts, with decreased IGF1 levels and expression of $I g f 1$ and $G h r$ in the skeletal muscle and liver. Germ-free mice under chronic undernutrition exhibit a resistance to GH signaling, as is the case in undernourished humans (Blanton et al. 2016b, Hoffman et al. 2017). Moreover, administration of recombinant IGF1 to germ-free mice normalizes weight and bone length, suggesting a direct impact of the gut microbiota on IGF1 secretion (Schwarzer et al. 2016).

Yan et al. confirmed the link between gut microbiota, IGF1 secretion and bone growth using germ-free mice colonized with the microbiota of conventional mice. Despite no difference in circulating GH when compared to germ-free mice, colonized mice had increased IGF1 secretion. Besides, the effects of the gut microbiota on bone turnover is age dependent. Indeed, after 1 month, the authors observed a decrease both in bone formation and resorption, associated to decreased trabecular bone mass and body weight. However, after 8 months, colonization resulted in increased bone formation and growth (Yan et al. 2016). In a similar manner, fecal microbiota transplantation in suckling pigs increases body weight and plasma IGF1, associated to increased presence of Lactobacillus sp. and Faecalibacterium sp. (Cheng et al. 2019).

The precise mechanisms underlying microbial stimulation of the somatotropic GH/IGF1 axis remain to be elucidated. Microbial by-products such as short- chain fatty acids (SCFAs), produced by the fermentation of dietary fibers, have been shown to modulate IGF1 and GH secretion (Wang et al. 2013, Yan et al. 2016). In vitro, SCFAs inhibit GH production in bovine anterior pituitary cells, through activation of the cAMP/PKA/ CREB pathway (Wang et al. 2013). Furthermore, Yan et al. showed that SCFAs, when co-administered with antibiotics to deplete the microbiota, resulted in elevation of IGF1 in serum, adipose tissue and liver (Yan et al. 2016). One way through which SCFAs may modulate the somatotropic axis is via the secretion of ghrelin, which is a GH secretagogue (Fig. 1). Administration of an oligofructose prebiotic (thus increasing SCFA secretion by the microbiota) results in decreased ghrelin levels in obese individuals (Parnell \& Reimer 2009). SCFAs also decrease ghrelin secretion in humans (Rahat-Rozenbloom et al. 2017). Furthermore, SCFAs, lactate and bacterial strains including Bifidobacterium and Lactobacillus are able to attenuate ghrelin-mediated signaling in an embryonic kidney cell line (Hek293a) stably expressing the ghrelin receptor (Torres-Fuentes et al. 2019).

Taken together, these data show that the gut microbiota is a potent positive regulator of GH and IGF1 signaling, stimulating host growth and development.

\section{Bidirectional relationship effect of GH and IGF1 on the gut microbiota}

A 2016 study by Chen et al. explored the role of GH/ IGF1 on the gut microbiota community using a female mouse model of anorexia nervosa (i.e. nutrient-restricted mice). When compared to controls, anorexic BALB/c mice showed decreased microbial diversity (Chen et al. 2016). When anorexic mice received subcutaneous injections of IGF1, this led to restoration of body weight, accompanied by normalization of the microbiota composition (Chen et al. 2016). Furthermore, specific deletion of IGF1 in intestinal epithelial cells in mice decreases proliferation of the intestinal epithelium, along with increased bacterial translocation to the mesenteric lymph node and liver, and alteration of the cecal microbiota of mice (Zheng et al. 2018).

A direct role of $\mathrm{GH}$ on the gut microbiota of mice came from the study of Ames dwarf mice, a mouse model of hypopituitarism with deficiencies in several pituitary hormones, including GH (Wiesenborn et al. 2020). Ames mice at 2 months of age exhibited increased Bacteroidetes/Firmicutes ratio, when compared to WT littermates. Unlike control mice, Ames mice show minor 
microbiota shifts when put on caloric restriction. The authors thus concluded that GH deficiency resulted in a distinct microbial composition. However, Ames mice have multiple hormone deficiencies (including TSH and prolactin), so it does not allow the study of GH specifically. Another study by Jensen et al. confirmed that GH signaling is associated with altered gut microbial composition. Two mouse models were generated: one with a full knockout of $\mathrm{GH}\left(\mathrm{GH}^{-/-}\right)$and one with ectopic expression of bovine transgenic GH (bGH) (Jensen et al. $2020 a$ ). Both mouse models exhibit altered microbiota composition when compared to littermate controls, in opposing directions. $\mathrm{GH}^{-/-}$mice have reduced abundance of proteobacteria, campylobacteria and actinobacteria, whereas bGH mice exhibit an increase in those phyla. In line with that, several bacterial metabolic functions, such as SCFA, folate and heme B biosynthesis, also correlate with the presence of GH. Finally, GH is associated with altered intestinal length and morphology.

Overall, these studies show that there is a bidirectional relationship between gut microbiota and GH/IGF1 signaling (Fig. 1).

\section{Gut microbiota and altered growth}

\section{Juvenile microbiota and malnutrition}

Nutrient deficiency, particularly dietary protein, leads to growth retardation during pregnancy (Gluckman \& Pinal 2003). Stunting (as defined in the introduction) is characterized by reduced secretion of IGF1, but increased GH levels characteristic of a GH resistant state. The 2020 Global Nutrition Report (https://globalnutritionreport. org/reports/2020-global-nutrition-report/) estimates that over 150 million children aged 0-59 months (representing $22.2 \%$ of children globally) are stunted. The first 1000 days of life are crucial for growth. After that time, stunting is usually irreversible and leads to growth delay in adulthood (Shrimpton et al. 2001).

Decreased microbial diversity and delayed microbiota maturation are well-described features of severe acute malnutrition (SAM) (Blanton et al. 2016a). Microbiota immaturity correlates with childhood stunting and wasting (Subramanian et al. 2014, Blanton et al. 2016b) and is only partially improved by nutritional intervention, suggesting that altered microbial composition may contribute to the low success rates of nutritional rehabilitation programs. Transplantation of stool from Malawian twins discordant for kwashiorkor (a severe form of protein malnutrition) into germ-free recipient mice maintained on a low-protein, low-fat diet showed that mice receiving fecal microbiota from severely malnourished children lose more weight that mice colonized with stool from healthy siblings (Smith et al. 2013).

Chen et al. studied the microbiota of stunted Bangladeshi children, who had not benefited from a nutritional intervention. Bacterial taxa from duodenal aspirates were negatively correlated with linear growth and positively correlated with inflammation. Moreover, colonization of germ-free mice with the duodenal aspirates led to enteropathy, showing a causal relationship between growth stunting and components of the duodenal microbiota (Chen et al. 2020).

\section{Microbiota-targeting therapies}

Despite evidence that malnutrition severely alters the microbial community of the gut, interventional trials using microbiota-targeting therapies have not shown any significant improvement on the growth phenotype. For instance, administration of a synbiotic product (containing several bacteria from the Lactobacillales order in combination with prebiotics) did not improve SAM outcomes in Malawian hospitalized children when compared to placebo treatment (Kerac et al. 2009). In the same manner, administration of a probiotic formula containing Lactocaseibacillus (formerly Lactobacillus) rhamnosus GG ATCC 53103 and Bifidobacterium animalis ssp. lactis Bb12 to children with SAM increases microbiota diversity but does not improve nutritional recovery and weight gain upon refeeding (Castro-Mejía et al. 2020).

The choice of the bacterial strains used in these trials was empiric (for a discussion on the matter, see Edwards et al. 2020). However, restoring the growth function upon severe malnutrition requires targeting an agent that goes beyond modification of the microbiota, which involves understanding the mechanisms underlying the interactions between the microbial strain(s) and the host.

Efforts have been made in this direction to identify growth-stimulating bacteria in preclinical models. Several studies have confirmed the capacity of a single bacteria to stimulate the GH/IGF1 somatotropic axis, a finding conserved among animal species as evolutionary distant as insects and mice (Shin et al. 2011, Storelli et al. 2011, Schwarzer et al. 2016). Particularly, administration of the specific strain of Lactobacillus plantarum WJL to germfree mice on a protein- and fat-depleted diet abrogates GH resistance and improves IGF1 and IGFBP3 secretion, resulting in overall growth (Schwarzer et al. 2016). Importantly, this phenotype is strain-specific, since a 
second strain of $L$. plantarum did not confer such effects on growth. Similarly, oral supplementation of three different strains of $L$. plantarum to broiler chickens exposed to heat stress led to improved growth performance and increased expression of hepatic Igf1 (Humam et al. 2019).

The main challenge to translating this approach to malnourished children is the incomplete knowledge of microbiota composition among large populations and of its variations between healthy and malnourished kids (who also differ in genetics, diets, and lifestyle). Additionally, probiotics-based approaches need to be monitored carefully, given that the immune system of severely malnourished children is constantly challenged.

Dietary approaches might thus represent a promising therapy to target the stunted microbiota. Gehrig et al. monitored metabolic parameters in healthy Bangladeshi children and those recovering from SAM, as they transitioned to moderate malnutrition with persistent microbiota immaturity. Diets were designed using pig and mouse models to drive the microbiota into a mature post-weaning state, expected to stimulate growth. These microbiota-directed complementary food (MDCF) prototypes were tested in gnotobiotic mice and piglets with immature microbiota (from children recovering from SAM). The authors determined the most efficient MDCF in a randomized double-blind controlled feeding trail, which drove plasma proteome profiles toward those of healthy children (Gehrig et al. 2019). In particular, this MDCF increased the abundance of age- and growthdiscriminatory microbial taxa. An ongoing clinical trial will determine if these microbiota changes translate into stimulation of growth in stunted children (Mostafa et al. 2020).

Another approach has been the study of the properties of human milk oligosaccharides (HMOs) in the context of juvenile growth. HMOs act as prebiotics that help colonization of the infant gut with bacterial taxa (such as Bifidobacterium spp.) associated to beneficial functions on growth and immune function (Charbonneau et al. 2016). It is noteworthy that HMOs are significantly depleted in breast milk of Malawian mothers with children suffering from SAM. Germ-free mice were colonized with a microbial community characteristic of stunted Malawian children and then fed with prototypic Malawian diet with or without supplementation with HMOs. Gnotobiotic mice fed HMOs showed increased lean mass gain, associated to increased bone volume and density. In a similarly designed study, Cowardin et al. colonized germfree mice with the microbiota of stunted infants and fed a diet mimicking that consumed by the microbiota donor.
Adding purified bovine sialylated milk oligosaccharides to the diet resulted in reduced osteoclastogenesis while increasing femoral trabecular bone volume and cortical thickness (Cowardin et al. 2019). Interestingly, the addition of milk oligosaccharides did not increase circulating IGF1, suggesting that stimulation of growth by the gut microbiota can also happen independently of GH/IGF1 pathway.

Such advances in the clinical literature regarding microbiota-directed therapies for malnutrition represent a promising strategy to tackle this worldwide problem. The first intention when treating malnutrition is refeeding but as pointed before, SAM can have long-lasting deleterious consequences despite nutritional restoration. Thus, understanding how the gut microbiota regulates growth and IGF1 secretion will help develop robust strategies with selected bacterial strains and/or prebiotics in the management of malnutrition and stunting.

\section{Conclusion}

The GH/IGF1 somatotropic axis is central during mammalian development through its influence on the growth of numerous organs. Studies in germ-free mice under normal and depleted diet have demonstrated the ability of the gut microbiota to directly modulate IGF1 circulating levels. The idea that a bidirectional relationship between the GH/IGF1 axis and the gut microbiota could exist emerged from studies in $\mathrm{GH}^{-/-}$mice demonstrating the ability of GH signaling to modulate the gut microbiota composition and intestinal physiology. Undernutrition has been associated with decreased microbial diversity and delayed microbiota maturation. Thus, microbiota interacts with both nutrition and the GH/IGF1 axis. Further research needs to be conducted to understand to what extend this tripartite relationship regulates systemic growth.

In the context of undernutrition, the first line of treatment should of course be refeeding with proper macronutrient balance. However, since interventional studies have shown this is not enough to fully counteract the deleterious effects of protein depletion in early life, microbiota-targeting therapies that allow not only microbiota maturation but also growth reestablishment could be promising treatments for undernourished children. Understanding the molecular mechanisms governing growth hormones-microbiota interactions using preclinical animal models is thus required. This would help identify promising probiotic candidates and therefore develop therapeutic strategies for stunted 
children. Despite a putative function of microbiotaderived short-chain fatty acids in inhibiting ghrelin secretion and thus GH production, the exact molecular mechanisms underlying the effects of microbiota on growth remain poorly understood. Notably, microbiota could stimulate growth independently from the GH/IGF1 axis through interactions with human milk oligosaccharides.

\section{Declaration of interest}

The authors declare that there is no conflict of interest that could be perceived as prejudicing the impartiality of this review.

\section{Funding}

F.D.V. is supported by a grant from La Fondation des Treilles. A.J. is supported by a doctoral grant from the French Ministry of Research. Research in F.L. lab is supported by Université de Lyon, ENS de Lyon and CNRS and funded by an FRM grant (Équipe FRM DEQ20180339196) and an ANR grant (ANR-18-CE15-0011-01).

\section{References}

Bartke A, Sun LY \& Longo V 2013 Somatotropic signaling: trade-offs between growth, reproductive development, and longevity. Physiological Reviews 93 571-598. (https://doi.org/10.1152/ physrev.00006.2012)

Blanton LV, Barratt MJ, Charbonneau MR, Ahmed T \& Gordon JI $2016 a$ Childhood undernutrition, the gut microbiota, and microbiotadirected therapeutics. Science 352 1533. (https://doi.org/10.1126/ science.aad9359)

Blanton LV, Charbonneau MR, Salih T, Barratt MJ, Venkatesh S, Ilkaveya O, Subramanian S, Manary MJ, Trehan I, Jorgensen JM, et al. $2016 \mathrm{~b}$ Gut bacteria that prevent growth impairments transmitted by microbiota from malnourished children. Science $\mathbf{3 5 1}$ add3311. (https://doi.org/10.1126/science.aad3311)

Burgus R, Ling N, Butcher M \& Guillemin R 1973 Primary structure of somatostatin, a hypothalamic peptide that inhibits the secretion of pituitary growth hormone. Proceedings of the National Academy of Sciences of the United States of America 70 684-688. (https://doi. org/10.1073/pnas.70.3.684)

Carro E, Señaris R, Considine RV, Casanueva FF \& Dieguez C 1997 Regulation of in vivo growth hormone secretion by leptin. Endocrinology 138 2203-2206. (https://doi.org/10.1210/ endo.138.5.5238)

Castro-Mejía JL, O’Ferrall S, Krych Ł, O'Mahony E, Namusoke H, Lanyero B, Kot W, Nabukeera-Barungi N, Michaelsen KF, Mølgaard C, et al. 2020 Restitution of gut microbiota in Ugandan children administered with probiotics (Lactobacillus rhamnosus GG and Bifidobacterium animalis subsp. lactis BB-12) during treatment for severe acute malnutrition. Gut Microbes 11 855-867. (https://doi.org/ 10.1080/19490976.2020.1712982)

Charbonneau MR, O'Donnell D, Blanton LV, Totten SM, Davis JCC, Barratt MJ, Cheng J, Guruge J, Talcott M, Bain JR, et al. 2016 Sialylated milk oligosaccharides promote microbiota-dependent growth in models of infant undernutrition. Cell 164 859-871. (https://doi.org/10.1016/j.cell.2016.01.024)

Chen J, Toyomasu Y, Hayashi Y, Linden DR, Szurszewski JH, Nelson H, Farrugia G, Kashyap PC, Chia N \& Ordog T 2016 Altered gut microbiota in female mice with persistent low body weights following removal of post-weaning chronic dietary restriction. Genome Medicine 8 103. (https://doi.org/10.1186/ s13073-016-0357-1)

Chen RY, Kung VL, Das S, Hossain MS, Hibberd MC, Guruge J, Mahfuz M, Begum SMKN, Rahman MM, Fahim SM, et al. 2020 Duodenal microbiota in stunted undernourished children with enteropathy. New England Journal of Medicine 383 321-333. (https:// doi.org/10.1056/NEJMoa1916004)

Cheng CS, Wei HK, Wang P, Yu HC, Zhang XM, Jiang SW \& Peng J 2019 Early intervention with faecal microbiota transplantation: an effective means to improve growth performance and the intestinal development of suckling piglets. Animal 13 533-541. (https://doi. org/10.1017/S1751731118001611)

Cowardin CA, Ahern PP, Kung VL, Hibberd MC, Cheng J, Guruge JL, Sundaresan V, Head RD, Barile D, Mills DA, et al. 2019 Mechanisms by which sialylated milk oligosaccharides impact bone biology in a gnotobiotic mouse model of infant undernutrition. Proceedings of the National Academy of Sciences of the United States of America $\mathbf{1 1 6}$ 11988-11996. (https://doi.org/10.1073/pnas.1821770116)

Delehaye-Zervas MC, Mertani H, Martini JF, Nihoul-Feketé C, Morel G \& Postel-Vinay MC 1994 Expression of the growth hormone receptor gene in human digestive tissue. Journal of Clinical Endocrinology and Metabolism 78 1473-1480. (https://doi.org/10.1210/ jcem.78.6.8200952)

Edwards PT, Kashyap PC \& Preidis GA 2020 Microbiota on biotics: probiotics, prebiotics, and Synbiotics to optimize growth and metabolism. American Journal of Physiology. Gastrointestinal and Liver Physiology 319 G382-G390. (https://doi.org/10.1152/ ajpgi.00028.2020)

Fazeli PK \& Klibanski A 2014 Determinants of GH resistance in malnutrition. Journal of Endocrinology 220 R57-R65. (https://doi. org/10.1530/JOE-13-0477)

Gehrig JL, Venkatesh S, Chang HW, Hibberd MC, Kung VL, Cheng J, Chen RY, Subramanian S, Cowardin CA, Meier MF, et al. 2019 Effects of microbiota-directed foods in gnotobiotic animals and undernourished children. Science 365. (https://doi.org/10.1126/ science.aau4732)

Gluckman PD \& Pinal CS 2003 Regulation of fetal growth by the somatotrophic axis. Journal of Nutrition $\mathbf{1 3 3}$ (Supplement 2) 1741S-1746S. (https://doi.org/10.1093/jn/133.5.1741S)

Hoffman DJ, Campos-Ponce M, Taddei CR \& Doak CM 2017 Microbiome, growth retardation and metabolism: are they related? Annals of Human Biology 44 201-207. (https://doi.org/10.1080/03014 460.2016.1267261)

Humam AM, Loh TC, Foo HL, Samsudin AA, Mustapha NM, Zulkifli I \& Izuddin WI 2019 Effects of feeding different postbiotics produced by Lactobacillus plantarum on growth performance, carcass yield, intestinal morphology, gut microbiota composition, immune status, and growth gene expression in broilers under heat stress. Animals 9 (https://doi.org/10.3390/ani9090644)

Jensen EA, Young JA, Jackson Z, Busken J, List EO, Carroll RK, Kopchick JJ, Murphy ER \& Berryman DE 2020a Growth hormone deficiency and excess alter the gut microbiome in adult male mice. Endocrinology 161. (https://doi.org/10.1210/endocr/bqaa026)

Jensen EA, Young JA, Mathes SC, List EO, Carroll RK, Kuhn J, Onusko M, Kopchick JJ, Murphy ER \& Berryman DE 2020b Crosstalk between the growth hormone/insulin-like growth factor-1 axis and the gut microbiome: a new frontier for microbial endocrinology. Growth Hormone \& IGF Research 53-54 101333. (https://doi. org/10.1016/j.ghir.2020.101333)

Karsenty G 2006 Convergence between bone and energy homeostases: leptin regulation of bone mass. Cell Metabolism 4 341-348. (https:// doi.org/10.1016/j.cmet.2006.10.008)

Kerac M, Bunn J, Seal A, Thindwa M, Tomkins A, Sadler K, Bahwere P \& Collins S 2009 Probiotics and prebiotics for severe acute malnutrition (PRONUT study): a double-blind efficacy randomised 
controlled trial in Malawi. Lancet 374 136-144. (https://doi. org/10.1016/S0140-6736(09)60884-9)

Kojima M, Hosoda H, Date Y, Nakazato M, Matsuo H \& Kangawa K 1999 Ghrelin is a growth-hormone-releasing acylated peptide from stomach. Nature $\mathbf{4 0 2}$ 656-660. (https://doi.org/10.1038/45230)

Mackie EJ, Tatarczuch L \& Mirams M 2011 The skeleton: a multifunctional complex organ: the growth plate chondrocyte and endochondral ossification. Journal of Endocrinology 211 109-121. (https://doi.org/10.1530/JOE-11-0048)

Mohan S \& Kesavan C 2012 Role of insulin-like growth factor-1 in the regulation of skeletal growth. Current Osteoporosis Reports $\mathbf{1 0}$ 178-186. (https://doi.org/10.1007/s11914-012-0100-9)

Mostafa I, Nahar NN, Islam MM, Huq S, Mustafa M, Barratt M, Gordon JI \& Ahmed T 2020 Proof-of-concept study of the efficacy of a microbiota-directed complementary food formulation (MDCF) for treating moderate acute malnutrition. BMC Public Health 20242. (https://doi.org/10.1186/s12889-020-8330-8)

Netchine I, Azzi S, Le Bouc Y \& Savage MO 2011 IGF1 molecular anomalies demonstrate its critical role in fetal, postnatal growth and brain development. Best Practice \& Research. Clinical Endocrinology \& Metabolism 25 181-190. (https://doi.org/10.1016/j.beem.2010.08.005)

Novince CM, Whittow CR, Aartun JD, Hathaway JD, Poulides N, Chavez MB, Steinkamp HM, Kirkwood KA, Huang E, Westwater C, et al. 2017 Commensal gut microbiota immunomodulatory actions in bone marrow and liver have catabolic effects on skeletal homeostasis in health. Scientific Reports 7 5747. (https://doi. org/10.1038/s41598-017-06126-x)

Papaconstantinou J 2009 Insulin/IGF-1 and ROS signaling pathway cross-talk in aging and longevity determination. Molecular and Cellular Endocrinology 299 89-100. (https://doi.org/10.1016/j. mce.2008.11.025)

Parnell JA \& Reimer RA 2009 Weight loss during oligofructose supplementation is associated with decreased ghrelin and increased peptide YY in overweight and obese adults. American Journal of Clinical Nutrition 89 1751-1759. (https://doi.org/10.3945/ajcn.2009.27465)

Poinsot P, Schwarzer M, Peretti N \& Leulier F 2018 The emerging connections between IGF1, the intestinal microbiome, Lactobacillus strains and bone growth. Journal of Molecular Endocrinology $\mathbf{6 1}$ T103-T113. (https://doi.org/10.1530/JME-17-0292)

Pradhan G, Samson SL \& Sun Y 2013 Ghrelin: much more than a hunger hormone. Current Opinion in Clinical Nutrition and Metabolic Care 16 619-624. (https://doi.org/10.1097/MCO.0b013e328365b9be)

Rahat-Rozenbloom S, Fernandes J, Cheng J, Gloor GB \& Wolever TMS 2017 The acute effects of inulin and resistant starch on postprandial serum short-chain fatty acids and second-meal glycemic response in lean and overweight humans. European Journal of Clinical Nutrition 71 227-233. (https://doi.org/10.1038/ejcn.2016.248)

Schwarzer M, Makki K, Storelli G, Machuca-Gayet I, Srutkova D, Hermanova P, Martino ME, Balmand S, Hudcovic T, Heddi A, et al. 2016 Lactobacillus plantarum strain maintains growth of infant mice during chronic undernutrition. Science 351 854-857. (https://doi. org/10.1126/science.aad8588)

Sharples AP, Hughes DC, Deane CS, Saini A, Selman C \& Stewart CE 2015 Longevity and skeletal muscle mass: the role of IGF signalling, the sirtuins, dietary restriction and protein intake. Aging Cell $\mathbf{1 4}$ 511-523. (https://doi.org/10.1111/acel.12342)

Shin SC, Kim SH, You H, Kim B, Kim AC, Lee KA, Yoon JH, Ryu JH \& Lee WJ 2011 Drosophila microbiome modulates host developmental and metabolic homeostasis via insulin signaling. Science $\mathbf{3 3 4}$ 670-674. (https://doi.org/10.1126/science.1212782)

Shrimpton R, Victora CG, de Onis M, Lima RC, Blössner M \& Clugston G 2001 Worldwide timing of growth faltering: implications for nutritional interventions. Pediatrics 107 E75. (https://doi. org/10.1542/peds.107.5.e75)

Smith MI, Yatsunenko T, Manary MJ, Trehan I, Mkakosya R, Cheng J, Kau AL, Rich SS, Concannon P, Mychaleckyj JC, et al. 2013 Gut microbiomes of Malawian twin pairs discordant for kwashiorkor. Science 339 548-554. (https://doi.org/10.1126/science.1229000)

Storelli G, Defaye A, Erkosar B, Hols P, Royet J \& Leulier F 2011 Lactobacillus plantarum promotes Drosophila systemic growth by modulating hormonal signals through TOR-dependent nutrient sensing. Cell Metabolism 14 403-414. (https://doi.org/10.1016/j. cmet.2011.07.012)

Subramanian S, Huq S, Yatsunenko T, Haque R, Mahfuz M, Alam MA, Benezra A, DeStefano J, Meier MF, Muegge BD, et al. 2014 Persistent gut microbiota immaturity in malnourished Bangladeshi children. Nature 510 417-421. (https://doi.org/10.1038/nature13421)

Torres-Fuentes C, Golubeva AV, Zhdanov AV, Wallace S, Arboleya S, Papkovsky DB, El Aidy S, Ross P, Roy BL, Stanton C, et al. 2019 Short-chain fatty acids and microbiota metabolites attenuate ghrelin receptor signaling. FASEB Journal 33 13546-13559. (https://doi. org/10.1096/fj.201901433R)

Wang JF, Fu SP, Li SN, Hu ZM, Xue WJ, Li ZQ, Huang BX, Lv QK, Liu JX \& Wang W 2013 Short-chain fatty acids inhibit growth hormone and prolactin gene transcription via cAMP/PKA/CREB signaling pathway in dairy cow anterior pituitary cells. International Journal of Molecular Sciences 14 21474-21488. (https://doi.org/10.3390/ ijms141121474)

Wiesenborn DS, Gálvez EJC, Spinel L, Victoria B, Allen B, Schneider A, Gesing A, Al-Regaiey KA, Strowig T, Schäfer KH, et al. 2020 The role of Ames dwarfism and calorie restriction on gut microbiota. Journals of Gerontology. Series A, Biological Sciences and Medical Sciences 75 e1e8. (https://doi.org/10.1093/gerona/glz236)

Yakar S \& Isaksson O 2016 Regulation of skeletal growth and mineral acquisition by the GH/IGF-1 axis: lessons from mouse models. Growth Hormone \& IGF Research 28 26-42. (https://doi.org/10.1016/j. ghir.2015.09.004)

Yakar S, Rosen CJ, Beamer WG, Ackert-Bicknell CL, Wu Y, Liu JL, Ooi GT, Setser J, Frystyk J, Boisclair YR, et al. 2002 Circulating levels of IGF-1 directly regulate bone growth and density. Journal of Clinical Investigation 110 771-781. (https://doi.org/10.1172/JCI15463)

Yan J, Herzog JW, Tsang K, Brennan CA, Bower MA, Garrett WS, Sartor BR, Aliprantis AO \& Charles JF 2016 Gut microbiota induce IGF-1 and promote bone formation and growth. Proceedings of the National Academy of Sciences of the United States of America $\mathbf{1 1 3}$ E7554-E7563. (https://doi.org/10.1073/pnas.1607235113)

Young JA, Jensen EA, Stevens A, Duran-Ortiz S, List EO, Berryman DE \& Kopchick JJ 2019 Characterization of an intestine-specific GH receptor knockout (IntGHRKO) mouse. Growth Hormone \& IGF Research 46-47 5-15. (https://doi.org/10.1016/j.ghir.2019.05.001)

Zheng Y, Song Y, Han Q, Liu W, Xu J, Yu Z, Zhang R \& Li N 2018 Intestinal epithelial cell-specific IGF1 promotes the expansion of intestinal stem cells during epithelial regeneration and functions on the intestinal immune homeostasis. American Journal of Physiology. Endocrinology and Metabolism 315 E638-E649. (https://doi. org/10.1152/ajpendo.00022.2018)
Received in final form 14 December 2020

Accepted 5 January 2021

Accepted Manuscript published online 8 January 2021 